\title{
THE DIRAC DELTA FUNCTION IN TWO SETTINGS OF REVERSE MATHEMATICS
}

\author{
SAM SANDERS AND KEITA YOKOYAMA
}

\begin{abstract}
The program of Reverse Mathematics $([18])$ has provided us with the insight that most theorems of ordinary mathematics are either equivalent to one of a select few logical principles, or provable in a weak base theory. In this paper, we study the properties of the Dirac delta function (3, 15]) in two settings of Reverse Mathematics. In particular, we consider the Dirac Delta Theorem, which formalizes the well-known property $\int_{\mathbb{R}} f(x) \delta(x) d x=f(0)$ of the Dirac delta function. We show that the Dirac Delta Theorem is equivalent to weak weak König's Lemma (see 23]) in classical Reverse Mathematics. This further validates the status of $W_{W K L_{0}}$ as one of the 'Big' systems of Reverse Mathematics. In the context of ERNA's Reverse Mathematics $([14]$ ), we show that the Dirac Delta Theorem is equivalent to the Universal Transfer Principle. Since the Universal Transfer Principle corresponds to WKL, it seems that, in ERNA's Reverse Mathematics, the principles corresponding to WKL and WWKL coincide. Hence, ERNA's Reverse Mathematics is actually coarser than classical Reverse Mathematics, although the base theory has lower first-order strength.
\end{abstract}

\section{INTRODUCTION}

Reverse Mathematics is a program in Foundations of Mathematics initiated around 1975 by Harvey Friedman ([4] and [5]) and developed intensely by Stephen Simpson and others. For an overview of the subject, we refer to [18 and 19]. The principal goal of Reverse Mathematics is to determine the minimal axiom system necessary to prove a particular theorem of ordinary mathematics. By now, it is well-known that large portions of mathematics (especially so in analysis) can be carried out in systems far weaker than ZFC, the 'usual' background theory for mathematics. Classifying theorems according to their logical strength reveals the following striking phenomenon: It turns out that, in many particular cases, if a mathematical theorem is proved from appropriately weak set existence axioms, then the axioms will be logically equivalent to the theorem ([18, Preface $]$ ). This recurring phenomenon is called the 'Main theme' of Reverse Mathematics (see e.g. 16]) and a good instance of it, is the following theorem from [18, p. 36].

1. Theorem (Reverse Mathematics for $\left.\mathrm{WKL}_{0}\right)$. Within $\mathrm{RCA}_{0}$, one can prove that Weak König's Lemma (WKL) is equivalent to each of the following mathematical statements:

1. The Heine-Borel lemma: every covering of $[0,1]$ by a sequence of open intervals has a finite subcovering.

2. Every covering of a compact metric space by a sequence of open sets has a finite subcovering.

3. Every continuous real-valued function on $[0,1]$ is bounded.

4. Every continuous real-valued function on $[0,1]$ is uniformly continuous.

5. Every continuous real-valued function on $[0,1]$ is Riemann integrable.

Ghent University, Department of Mathematics, Krijgslaan 281, B-9000 Gent (Belgium)

Tohoku University, Mathematical Institute, 980-8578 Sendai (Japan)

E-mail addresses: sasander@cage.ugent.be, y-keita@math.tohoku.ac.jp. 
6. The maximum principle: every continuous real-valued function on $[0,1]$ is bounded, or (equivalently) has a supremum or (equivalently) attains its maximum.

7. The Peano existence theorem for ordinary differential equations.

8. Gödel's completeness theorem: every at most countable consistent set of sentences in the predicate calculus has a countable model.

9. Every countable commutative ring has a prime ideal.

10. Every countable field (of characteristic 0) has a unique algebraic closure.

11. Every countable formally real field is orderable.

12. Every countable formally real field has a (unique) real closure.

13. Brouwer's fixed point theorem.

14. The separable Hahn-Banach theorem: if $f$ is a bounded linear functional on a subspace of a separable Banach space, and if $\|f\| \leq 1$, then $f$ has an extension $\tilde{f}$ to the whole space such that $\|\tilde{f}\| \leq 1$.

Here, the theory $\mathrm{RCA}_{0}$ is the 'base theory' of Reverse Mathematics. This theory is strong enough to formalize the notion of e.g. continuous $\mathbb{R} \rightarrow \mathbb{R}$-function, but $\mathrm{RCA}_{0}$ is too weak to prove any of the items in the previous theorem. These require WKL, which is the statement that every infinite 0-1-labeled tree has an infinite path. The theory $\mathrm{WKL}_{0}$ is defined as $\mathrm{RCA}_{0}$ plus WKL and is strictly stronger than $\mathrm{RCA}_{0}$. By the previous theorem, many theorems of ordinary mathematics are equivalent to $\mathrm{WKL}_{0}$, given $\mathrm{RCA}_{0}$. There are three more theories $\left(\mathrm{ACA}_{0}, \mathrm{ATR}_{0}\right.$ and $\left.\Pi_{1}^{1}-\mathrm{CA}_{0}\right)$ with the latter property (see 18 for details). The latter theories, together with $\mathrm{RCA}_{0}$ and $\mathrm{WKL}_{0}$, are called the 'Big Five'. Although there are infinitely many nonequivalent logical theories, most theorems of ordinary mathematics (with few exceptions) are equivalent to one of the Big Five, or provable in $\mathrm{RCA}_{0}$.

In 23], Simpson and Yu introduce 'weak weak König's Lemma' (WWKL). This principle intuitively states that if a 0-1-labeled tree has no infinite path, then the probability of having a path of length $n$ in the tree becomes zero as $n$ tends towards infinity (See Principle 7). As the latter is a weakened version of WKL, we expect weakened versions of the items in Theorem 1 to be equivalent to WWKL. For instance, consider item 5 from Theorem 1. By Theorem 10 below, we see that the statement every continuous bounded real-valued function on $[0,1]$ is Riemann integrable is equivalent to WWKL. Moreover, several principles of measure theory and Fourier analysis are equivalent to WWKL (see e.g. 1, 18, 22]). Thus, it seems plausible to extend the 'Big Five' with $\mathrm{WWKL}_{0}$ (which is $\mathrm{RCA}_{0}$ plus WWKL) to the 'Big Six'. In Section 2, we provide further evidence to support such an extension. In particular, we consider the Dirac Delta function (see [3, 15]) in the context of classica $]^{1}$ Reverse Mathematics. The Dirac Delta Theorem (DDT for short) is the formalization of the Dirac delta function's essential property $\int_{\mathbb{R}} f(x) \delta(x) d x=f(0)$. In section 2 , we prove that a version of DDT is equivalent to WWKL. Furthermore, we observe that DDT is not a weakened version of a theorem equivalent to WKL. Thus, we believe this result to be important evidence for the acceptance of $\mathrm{WWKL}_{0}$ as one of the 'Big' systems of Reverse Mathematics.

In Section 3, we consider the Dirac Delta Theorem in the context of ERNA's Reverse Mathematics. The latter was introduced in [14] and we now briefly sketch its main features. In 6] and [18, X.4.3], it is suggested to reduce the first-order strength of the base theory of Reverse Mathematics to roughly $I \Delta_{0}+$ exp. In [14, Reverse Mathematics was developed in the theory ERNA (see [9, 20]), a nonstandard extension of $I \Delta_{0}+$ exp. The main result of [14] is the following

\footnotetext{
${ }^{1}$ We use 'classical Reverse Mathematics' to refer to the usual subsystems of second-order arithmetic from 18 .
} 
theorem; it contains several statements, translated from Theorem 1 and 18 , Chapter IV] into ERNA's language, while preserving equivalence. For the definitions, see 14 and Section 3 below.

2. Theorem (Reverse Mathematics for ERNA $+\Pi_{1}$-TRANS). The theory ERNA proves the equivalence between $\Pi_{1}$-TRANS and each of the following theorems concerning near-standard functions:

1. Every S-continuous function on $[0,1]$, or on any interval, is bounded.

2. Every $S$-continuous function on $[0,1]$, or on any interval, is continuous there.

3. Every S-continuous function on $[0,1]$, or on any interval, is Riemann integrable.

4. Weierstrass' theorem: every S-continuous function on $[0,1]$, or on any interval, has, or attains a supremum, up to infinitesimals.

5. The uniform Brouwer fixed point theorem: every S-continuous function $\phi:[0,1] \rightarrow[0,1]$ has a fixed point up to infinitesimals of arbitrary depth.

6. The first fundamental theorem of calculus.

7. The Peano existence theorem for ordinary differential equations.

8. The Cauchy completeness, up to infinitesimals, of ERNA's field.

9. Every $S$-continuous function on $[0,1]$ has a modulus of uniform continuity.

10. The Weierstrass approximation theorem.

A common feature of the items in the previous theorem is that strict equality has been replaced with $\approx$, i.e. equality up to infinitesimals. This seems the price to be paid for 'pushing down' into ERNA the theorems equivalent to WKL. For instance, item 7 guarantees that there exists $\phi(x)$ such that $\phi^{\prime}(x) \approx f(x, \phi(x))$, i.e. a solution, up to infinitesimals, of the differential equation $y^{\prime}=f(x, y)$. However, in general, there is no function $\psi(x)$ such that $\psi^{\prime}(x)=f(x, \psi(x))$. In this way, we say that the Reverse Mathematics of ERNA + $\Pi_{1}$-TRANS is a 'copy up to infinitesimals' of the Reverse Mathematics of $\mathrm{WKL}_{0}$. Moreover, it can be shown that ERNA $+\Pi_{1}$-TRANS is conservative over $B \Sigma_{2}$. The latter is $\Pi_{3}$-conservative over $I \Sigma_{1}$, the first-order theory of $\mathrm{WKL}_{0}$.

Below, we prove that ERNA's version of DDT is equivalent to the Universal Transfer Principle $\Pi_{1}$-TRANS. Together with Theorems 10, 12, and 31, this suggests that WKL and WWKL coincide in the setting of ERNA's Reverse Mathematics. We also consider the Heaviside function $H(x)$ and its main distributional property $\frac{d H}{d x}=\delta(x)$.

\section{The Dirac Delta in classical Reverse Mathematics}

In this section, we consider the Dirac delta function in second-order arithmetic, i.e. in classical Reverse Mathematics. We prove that $\mathrm{WWKL}_{0}$ is essentially needed to handle the Dirac delta function. Indeed, by Theorem 12, a version of the Dirac Delta Theorem is equivalent to weak weak König's Lemma, within $\mathrm{RCA}_{0}$.

2.1. Preliminaries. For the rest of this section, we adopt the usual definition for continuous functions on $\mathbb{R}$ in second-order arithmetic (see $[18$, Chapter II]). The notions of modulus of uniform continuity and derivative are also defined as usual in second-order arithmetic (see also [22]). We first define $\mathrm{C}^{1}, \mathrm{C}^{r}$ and $\mathrm{C}^{\infty}$-functions in $\mathbb{R}$.

3. Definition $\left(\mathrm{C}^{1}-, \mathrm{C}^{r}\right.$ - and $\mathrm{C}^{\infty}$-functions). The following definitions are made in $\mathrm{RCA}_{0}$.

1. Let $U$ be an open subset of $\mathbb{R}$, and let $f, f^{\prime}$ be continuous functions from $U$ to $\mathbb{R}$. Then a pair $\left(f, f^{\prime}\right)$ is said to be of $\mathrm{C}^{1}$ if and only if

$$
\forall x \in U \lim _{u \rightarrow x} \frac{f(u)-f(x)}{u-x}=f^{\prime}(x) .
$$


2. Let $U$ be an open subset of $\mathbb{R}$, and let $\left\{f^{(n)}\right\}_{n \leq r}$ be a finite sequence of continuous functions from $U$ to $\mathbb{R}$. Then $\left\{f^{(n)}\right\}_{n \leq r}$ is said to be of $\mathrm{C}^{r}$ if and only if for any $n$ less than $r$, $\left(f^{(n)}, f^{(n+1)}\right)$ is of $\mathrm{C}^{1}$.

3. Let $U$ be an open subset of $\mathbb{R}$, and let $\left\{f^{(n)}\right\}_{n \in \mathbb{N}}$ be an infinite sequence of continuous functions from $U$ to $\mathbb{R}$. Then $\left\{f^{(n)}\right\}_{n \in \mathbb{N}}$ is said to be of $\mathrm{C}^{\infty}$ if and only if for any $r \in \mathbb{N}$, $\left\{f^{(n)}\right\}_{n \leq r}$ is of $\mathrm{C}^{r}$.

4. Notation. We usually write $f$ for $f^{(0)}$ when $\left\{f^{(n)}\right\}_{n \leq r}$ is of $\mathrm{C}^{r}$ or $\left\{f^{(n)}\right\}_{n \in \mathbb{N}}$ is of $\mathrm{C}^{\infty}$. If $\left(f, f^{\prime}\right)$ is of $\mathrm{C}^{1},\left\{f^{(n)}\right\}_{n \leq r}$ is of $\mathrm{C}^{r}$ or $\left\{f^{(n)}\right\}_{n \in \mathbb{N}}$ is of $\mathrm{C}^{\infty}, f$ is said to be of $\mathrm{C}^{1}, \mathrm{C}^{r}$, or $\mathrm{C}^{\infty}$, respectively.

Note that we cannot ensure the existence of the derivative function $f^{\prime}$ even if $f$ is continuously differentiable at each point. Indeed, this requires $\mathrm{ACA}_{0}$ (see [22, Theorem 3.8]).

We recall the definition of Riemann integral and effective integrability in $\mathrm{RCA}_{0}$.

5. Definition (Riemann integral in $\mathrm{RCA}_{0}$ ). Let $f$ be a continuous function from $[a, b]$ to $\mathbb{R}$.

1. The set $\Delta=\left\{a=x_{0} \leq \xi_{1} \leq x_{1} \leq \cdots \leq \xi_{n} \leq x_{n}=b\right\}$ is called a partition of $[a, b]$ and the number $|\Delta|=\max \left\{x_{k}-x_{k-1} \mid 1 \leq k \leq n\right\}$ is called the mesh of $\Delta$.

2. The sum $S_{\Delta}(f)=\sum_{k=1}^{n} f\left(\xi_{k}\right)\left(x_{k}-x_{k-1}\right)$ is called a Riemann sum.

3. The limit $\int_{a}^{b} f(x) d x=\lim _{|\Delta| \rightarrow 0} S_{\Delta}(f)$, if it exists, is called the Riemann integral.

6. Definition. The following definition is made in $\mathrm{RCA}_{0}$. A function $f$ is effectively integrable if there exists $h: \mathbb{N} \rightarrow \mathbb{N}$ such that for any $n \in \mathbb{N}$ and for any partitions $\Delta_{1}, \Delta_{2}$ of $[a, b]$,

$$
\left|\Delta_{1}\right|<\frac{2^{-h(n)}}{b-a} \wedge\left|\Delta_{2}\right|<\frac{2^{-h(n)}}{b-a} \rightarrow\left|S_{\Delta_{1}}(f)-S_{\Delta_{2}}(f)\right|<2^{-n+1} .
$$

For effectively integrable functions, We can prove the usual 'integration by parts' formulas and other basic calculus results in the standard way within $\mathrm{RCA}_{0}$. On the other hand, it can be shown, with substantial effort, that $\mathrm{RCA}_{0}$ proves that the existence of the Riemann integral implies effective integrability (see 22]).

We now recall weak weak König's lemma

7. Principle (WWKL). If $T$ is a subtree of $2^{\mathbb{N}}$ with no infinite path, then

$$
\lim _{n \rightarrow \infty} \frac{|\{\sigma \in T \mid \operatorname{lh}(\sigma)=n\}|}{2^{n}}=0 .
$$

The theory $\mathrm{WWKL}_{0}$ is $\mathrm{RCA}_{0}$ plus WWKL. By Theorem 1. $\mathrm{WKL}_{0}$ is exactly what is required to integrate a continuous function over a compact interval. However, to integrate bounded functions, we only need $\mathrm{WWKL}_{0}$. Note that within $\mathrm{RCA}_{0}$, we can easily show that the existence of a modulus of uniform continuity implies the effective integrability, but the converse is not true even if a function is bounded.

8. Lemma. The following assertions are equivalent over $\mathrm{RCA}_{0}$.

1. $W W K L_{0}$.

2. Let $\left\langle B\left(a_{k}, r_{k}\right) \mid k \in \mathbb{N}\right\rangle$ be a sequence of rational open balls such that $[0,1] \subseteq \bigcup_{k \in \mathbb{N}} B\left(a_{k}, r_{k}\right)$. For any $\varepsilon>0$, there is $i \in \mathbb{N}$ and a finite sequence of open intervals $\left\{\left(c_{j}, d_{j}\right)\right\}_{j \leq l}$ such that,

$$
[0,1] \subseteq \bigcup_{k<i} B\left(a_{k}, r_{k}\right) \cup \bigcup_{j \leq l}\left(c_{j}, d_{j}\right) \text { and } \sum_{j \leq l}\left|d_{j}-c_{j}\right|<\varepsilon .
$$


3. Let $\left\langle T_{n} \mid n \in \mathbb{N}\right\rangle$ be a sequence of subtrees of $2^{\mathbb{N}}$. If each of $T_{n}$ has no infinite path, then there is a double sequence of rational numbers $\left\langle p_{n k} \mid n \in \mathbb{N}, k \in \mathbb{N}\right\rangle$ such that for any $n \in \mathbb{N}$,

$$
\left|\left\{\sigma \in T_{k} \mid \operatorname{lh}(\sigma)=n\right\}\right| \leq p_{n k} 2^{n} \wedge \lim _{k \rightarrow \text { infty }} p_{n k}=0 .
$$

4. Let $\left\langle B\left(a_{n k}, r_{n k}\right) \mid n \in \mathbb{N}, k \in \mathbb{N}\right\rangle$ be a double sequence of rational open balls such that $[0,1] \subseteq \bigcup_{k \in \mathbb{N}} B\left(a_{n k}, r_{n k}\right)$ for all $n \in \mathbb{N}$. There is a double sequence of finite sequences of open intervals $\left\langle\left\{\left(c_{n i j}, d_{n i j}\right)\right\}_{j \leq l_{n i}} \mid n \in \mathbb{N}, i \in \mathbb{N}\right\rangle$ such that, for any $n \in \mathbb{N}$,

$$
[0,1] \subseteq \bigcup_{k<i} B\left(a_{n k}, r_{n k}\right) \cup \bigcup_{j \leq l_{n i}}\left(c_{n i j}, d_{n i j}\right) \text { and } \lim _{i \rightarrow \infty} \sum_{j \leq l_{n i}}\left|d_{n i j}-c_{n i j}\right|=0 .
$$

Proof. $1 \leftrightarrow 2$ is just the restatement of [1, Theorem 3.3]. To show $1 \rightarrow 3$, define $p_{n k}=\mid\left\{\sigma \in T_{k} \mid\right.$ $\operatorname{lh}(\sigma)=n\} \mid / 2^{n} .3 \rightarrow 1$ is trivial. $3 \leftrightarrow 4$ is just an easy generalization of $1 \leftrightarrow 2$.

9. Remark. Lemma 8 shows that a sequential version of the weak Heine/Borel theorem (item 4) corresponding to WWKL is provable from a trivial sequential version of WWKL (item 3). On the other hand, the following stronger sequential version of WWKL is equivalent to $\mathrm{WKL}_{0}$ over $R C A_{0}$;

Let $\left\langle T_{n} \mid n \in \mathbb{N}\right\rangle$ be a sequence of subtrees of $2^{\mathbb{N}}$ such that $\lim _{n \rightarrow \infty}\left|\left\{\sigma \in T_{n} \mid \operatorname{lh}(\sigma)=k\right\}\right| / 2^{k}>$ 0 for all $k \in \mathbb{N}$. There is a sequence $\left\langle f_{n} \mid n \in \mathbb{N}\right\rangle$ such that each $f_{n}$ is a path of $T_{n}$.

This is related to the results of Sakamoto and Yamazaki in $[12$.

10. Theorem. The following assertions are equivalent over $\mathrm{RCA}_{0}$.

1. WWKL $L_{0}$.

2. Every bounded continuous function on $[a, b]$ is (effectively) Riemann integrable.

Proof. We first show $1 \rightarrow 2$. We reason within $W W K L_{0}$. Let $f$ be a bounded continuous function on $[a, b]$. For details concerning the coding of continuous functions in $\mathrm{RCA}_{0}$, we refer to [18, II.6.1]. Without loss of generality, we can assume $[a, b]=[0,1]$ and $f(x) \in[-1,1]$ for $x \in[0,1]$. Let $F \subseteq \mathbb{N} \times \mathbb{Q} \times \mathbb{Q}^{+} \times \mathbb{Q} \times \mathbb{Q}^{+}$be a code for $f$. Define the $\Sigma_{1}^{0}$-formula $\varphi(n, a, r)$ as

$$
a \in \mathbb{Q} \wedge r \in \mathbb{Q}^{+} \wedge(\exists b \in \mathbb{Q})\left(\exists s \in \mathbb{Q}^{+}\right)\left[(a, 2 r) F(b, s) \wedge s<2^{-n-2}\right] .
$$

Now, using $\Delta_{1}^{0}$-comprehension, define the sequence $\left\{\left(a_{n k}, r_{n k}\right)\right\}_{k \in \mathbb{N}, n \in \mathbb{N}}$ such that

$$
(\forall n)(\forall a)(\forall r)\left[\varphi(n, a, r) \leftrightarrow(\exists k)\left[(a, r)=\left(a_{n k}, r_{n k}\right)\right]\right] .
$$

Note that $[0,1] \subseteq \bigcup_{k \in \mathbb{N}} B\left(a_{n k}, r_{n k}\right)$ for all $n \in \mathbb{N}$. Thus, by Lemma 8 there exists a double sequence of finite sequences of open intervals $\left\langle\left\{\left(c_{n i j}, d_{n i j}\right)\right\}_{j \leq l_{n i}} \mid n \in \mathbb{N}, i \in \mathbb{N}\right\rangle$ such that for any $n \in \mathbb{N}$,

$$
\begin{aligned}
& {[0,1] \subseteq \bigcup_{k<i} B\left(a_{n k}, r_{n k}\right) \cup \bigcup_{j \leq l_{n i}}\left(c_{n i j}, d_{n i j}\right),} \\
& \lim _{i \rightarrow \infty} \sum_{j \leq l_{n i}}\left|d_{n i j}-c_{n i j}\right|=0 .
\end{aligned}
$$

By the above, we can define a sequence $\left\{\hat{i}_{n}\right\}_{n \in \mathbb{N}}$ such that $\sum_{j \leq l_{n \hat{i}_{n}}}\left|d_{n \hat{i}_{n} j}-c_{n \hat{i}_{n} j}\right|<2^{-n-2}$ and put $\hat{l}_{n}:=l_{n \hat{i}_{n}}$. Finally, define the function $h$ as

$$
h(n):=\min \left\{q \in \mathbb{N} \mid 2^{-q}<\min \left\{r_{n k} \mid k \leq \hat{l}_{n}\right\}\right\} .
$$

Then, we can show that for any $n \in \mathbb{N}$ and for any partitions $\Delta_{1}, \Delta_{2}$ of $[0,1]$, we have

$$
\left|\Delta_{1}\right|<2^{-h(n)} \wedge\left|\Delta_{2}\right|<2^{-h(n)} \rightarrow\left|S_{\Delta_{1}}(f)-S_{\Delta_{2}}(f)\right|<2^{-n+1} .
$$

This implies that $f$ is (effectively) Riemann integrable. 
To show (1), we only need to show the following: for any $n \in \mathbb{N}$ and for any partitions $\Delta, \Delta^{\prime}$ of $[0,1]$, if $|\Delta|<2^{-h(n)}$ and $\Delta^{\prime}$ is a refinement of $\Delta$, then,

$$
\left|S_{\Delta}(f)-S_{\Delta^{\prime}}(f)\right|<2^{-n} .
$$

(Taking a common refinement of $\Delta_{1}$ and $\Delta_{2}$, (2) implies (1) immediately.) Fix $n \in \mathbb{N}$. Let $\Delta=\left\{0=x_{0}<x_{1}<\cdots<x_{N+1}=1\right\}$ be a partition of $[0,1]$ such that $|\Delta|<2^{-h(n)}$, and let $\Delta^{\prime}$ be its refinement. Put $\delta_{m}:=\left[x_{m}, x_{m+1}\right]$. To show (2), we only need to show that for any $\left\{\xi_{m}\right\}_{m \leq N},\left\{\xi_{m}^{\prime}\right\}_{m \leq N}$ such that $\xi_{m}, \xi_{m}^{\prime} \in \delta_{m}$, we have

$$
\sum_{m=0}^{N}\left|f\left(\xi_{m}\right)-f\left(\xi_{m}^{\prime}\right)\right|\left(x_{m+1}-x_{m}\right)<2^{-n}
$$

Define $I \subseteq\{0, \cdots N\}$ as

$$
I=:\left\{m \leq N \mid \delta_{m} \cap \bigcup_{k<\hat{i}_{n}} B\left(a_{n k}, r_{n k}\right)=\emptyset\right\}
$$

Then,

$$
\bigcup_{m \in I} \delta_{m} \subseteq[0,1] \backslash \bigcup_{k<\hat{i}_{n}} B\left(a_{n k}, r_{n k}\right)
$$

thus,

$$
\sum_{m \in I}\left(x_{m+1}-x_{m}\right) \leq \sum_{j \leq \hat{l}_{n}}\left|d_{n \hat{i}_{n} j}-c_{n \hat{i}_{n} j}\right| .
$$

On the other hand, if $m \notin I$, then, by the definition of $h$, we have $\xi_{m}, \xi_{m}^{\prime} \in \delta_{m} \subseteq B\left(a_{n k}, 2 r_{n k}\right)$. Thus, by the definition of $\left\{\left(a_{n k}, r_{n k}\right)\right\}_{n \in \mathbb{N}, k \in \mathbb{N}}$, we have $\left|f\left(\xi_{m}\right)-f\left(\xi_{m}^{\prime}\right)\right|<2^{-n-1}$ for all $m \notin I$. Therefore,

$$
\begin{aligned}
& \sum_{m=0}^{N}\left|f\left(\xi_{m}\right)-f\left(\xi_{m}^{\prime}\right)\right|\left(x_{m+1}-x_{m}\right) \\
\leq & \sum_{m \in I} 2\left(x_{m+1}-x_{m}\right)+\sum_{m \notin I} 2^{-n-1}\left(x_{m+1}-x_{m}\right) \\
\leq & 2 \sum_{j \leq l_{n \hat{i}_{n}}}\left|d_{n \hat{i}_{n} j}-c_{n \hat{i}_{n} j}\right|+2^{-n-1} \\
< & 2^{-n} .
\end{aligned}
$$

This completes the proof of $1 \rightarrow 2$.

To show $2 \rightarrow 1$, we adopt the following notation. For a tree $T \subseteq 2^{<\mathbb{N}}$, define a set $S_{T} \subseteq 2^{<\mathbb{N}}$ and $\lambda_{n}^{T} \in \mathbb{N}$ as

$$
\begin{aligned}
S_{T} & :=\left\{\sigma \in 2^{<\mathbb{N}} \mid \sigma \notin T \wedge \forall \tau \subseteq \sigma(\tau \neq \sigma \rightarrow \tau \in T)\right\} ; \\
\lambda_{n}^{T} & :=|\{\sigma \in T \mid \operatorname{lh}(\sigma)=n\}| .
\end{aligned}
$$

For a finite sequence $\sigma \in 2^{<\mathbb{N}}$, define $a_{\sigma}, b_{\sigma} \in \mathbb{Q}$ as

$$
\begin{aligned}
& a_{\sigma}:=\sum_{i<\operatorname{lh}(\sigma)} \frac{\sigma(i)}{2^{i+1}} ; \\
& b_{\sigma}:=a_{\sigma}+\frac{1}{2^{\ln (\sigma)}} .
\end{aligned}
$$

Thus, if $\sigma, \tau \in S_{T}$, then, $b_{\tau} \leq a_{\sigma}$ or $b_{\sigma} \leq a_{\tau}$. Note that a tree $T$ has a path if and only if $[0,1] \nsubseteq \bigcup_{\sigma \in S_{T}}\left[a_{\sigma}, b_{\sigma}\right]$. 
Now, we show $\neg 1 \rightarrow \neg 2$. We reason within RCA $A_{0}$. Assume $\neg$ WWKL. Then, there exists a number $q>0$ and a tree $T$ which has no path such that

$$
(\forall n \in \mathbb{N})\left(\lambda_{n}^{T} / 2^{n}>q\right) .
$$

Since $[0,1] \subseteq \bigcup_{\sigma \in S_{T}}\left[a_{\sigma}, b_{\sigma}\right]$, we can define a continuous function $f$ from $[0,1]$ to $[0,1]$ as

$$
f(x):= \begin{cases}\frac{x-a_{\sigma}}{c_{\sigma}-a_{\sigma}} & x \in\left[a_{\sigma}, c_{\sigma}\right] \wedge \sigma \in S_{T}, \\ \frac{b_{\sigma}-x}{b_{\sigma}-c_{\sigma}} & x \in\left[c_{\sigma}, b_{\sigma}\right] \wedge \sigma \in S_{T}\end{cases}
$$

where $c_{\sigma}:=\left(b_{\sigma}+a_{\sigma}\right) / 2$. To prove the continuity of $f$, note that for any $\sigma \in S_{T}$ such that $0<a_{\sigma}<b_{\sigma<1}$, we can effectively find $\tau, \tau^{\prime} \in S_{T}$ such that $b_{\tau}=a_{\sigma}$ and $b_{\sigma}=a_{\tau^{\prime}}$ since $T$ does not have a path. Thus, we can find a code for a continuous function $f$. See also the proof of [18, Theorem IV.2.3].

We show that the function $f(x)$ is not Riemann integrable. Define partitions $\Delta_{k}$ of $[0,1]$ as

$$
\Delta_{k}:=\left\{0 \leq \frac{1}{2^{k}} \leq \frac{2}{2^{k}} \leq \cdots \leq \frac{2^{k}-1}{2^{k}} \leq 1\right\}=\left\{\left[a_{\eta}, b_{\eta}\right] \mid \eta \in 2^{<\mathbb{N}} \wedge \operatorname{lh}(\eta)=k\right\} .
$$

Note that we can easily define $M_{\sigma}:=\max \left\{f(x) \mid x \in\left[a_{\sigma}, b_{\sigma}\right]\right\}$ and $m_{\sigma}:=\min \left\{f(x) \mid x \in\left[a_{\sigma}, b_{\sigma}\right]\right\}$. We show that, for any $k \in \mathbb{N}$,

$$
\sum_{\eta \in 2^{<N} \wedge \operatorname{lh}(\eta)=k}\left(M_{\eta}-m_{\eta}\right) 2^{-k}>q .
$$

If $\eta \in T$, there exists $\sigma \in S_{T}$ such that $\sigma \supseteq \eta$, implying $\left[a_{\eta}, b_{\eta}\right] \supseteq\left[a_{\sigma}, b_{\sigma}\right]$. Therefore, $\eta \in T$ implies $M_{\eta}-m_{\eta}=1$. Hence, for any $k \in \mathbb{N}$,

$$
\sum_{\eta \in 2^{<\sim \wedge} \wedge \operatorname{lh}(\eta)=k}\left(M_{\eta}-m_{\eta}\right) 2^{-k} \geq \sum_{\eta \in T \wedge \operatorname{lh}(\eta)=k} 2^{-k} \geq \lambda_{n}^{T} 2^{-n}>q .
$$

This completes the proof of $2 \rightarrow 1$.

2.2. The Dirac delta function. In this subsection, we consider the Dirac delta function. Within $\mathrm{RCA}_{0}$, we first define the Dirac delta functional $u$ on a class of $C^{k}$-functions as $u(f)=f(0)$ for a $C^{k}$-function $f$. Moreover, for $i \leq k$, we define the $i$-th derivative $u^{(i)}$ as $u^{(i)}(f)=(-1)^{i} f^{(i)}(0)$.

Within $\mathrm{RCA}_{0}$, these functionals can be considered as continuous linear operator on an appropriate functional space in the following sense. For $k \in \mathbb{N}$, let $C_{0}^{k}(\mathbb{R})$ be the class of all functions $f$ on $\mathbb{R}$ which satisfy the following:

- $f$ is of $\mathrm{C}^{k}$ and for any $i \leq k, f^{(i)}$ has a modulus of uniform continuity,

- $f$ has a bounded support, i.e. $(\exists K \in \mathbb{N})(\forall x \in \mathbb{R})(|x| \geq K \rightarrow f(x)=0)$.

Imitating [18, Exercise IV.2.13], we can consider $C_{0}^{k}(\mathbb{R})$ as a separable Banach space within RCA . Then, we define the continuous linear operator $u: C_{0}^{k}(\mathbb{R}) \rightarrow \mathbb{R}$ as $u(f)=f(0)$. Moreover, for $i \leq k$, we can define the $i$-th derivative $u^{(i)}: C_{0}^{k}(\mathbb{R}) \rightarrow \mathbb{R}$ (as a distribution) as $u^{(i)}(f)=(-1)^{i} f^{(i)}(0)$. Here, $v$ is said to be the derivative of $u$ if $v(g)=-u\left(g^{\prime}\right)$ for any $g \in \cap_{k} C_{0}^{k}(\mathbb{R})$. However, within $\mathrm{RCA}_{0}$, a $\mathrm{C}^{k}$-function $f$ may not be an element of $C_{0}^{k}(\mathbb{R})$, even if it has a bounded support. So, we will consider two versions of the Dirac delta function, on $C_{0}^{k}(\mathbb{R})$ and on all $C^{k}$-functions.

Now we consider the Dirac delta function theorem, i.e. a limit representation of the Dirac delta function. We fix a smooth function $\eta: \mathbb{R} \rightarrow \mathbb{R}$ which satisfies the following:

- $\eta$ is of $\mathrm{C}^{\infty}$ and for any $i \in \mathbb{N}, \eta^{(i)}$ has a modulus of uniform continuity,

- $\operatorname{supp}(\eta) \subseteq[-1,1]$, i.e. $\eta(x)=0$ for any $x \notin(-1,1)$,

- $\int_{\mathbb{R}} \eta(x) d x=1$ and $\eta(x)>0$ for $x \in\left[-\frac{1}{2}, \frac{1}{2}\right]$. 
Define a sequence of smooth functions $\left\{\delta_{n}\right\}_{n \in \mathbb{N}}$ as $\delta_{n}(x)=2^{n} \eta\left(2^{n} x\right)$. Then, the limit of $\left\{\delta_{n}\right\}_{n \in \mathbb{N}}$ can be considered as the Dirac delta function $\delta(x)$ in the following sense.

11. Theorem. The following is provable within $\mathrm{RCA}_{0}$. Let $k \in \mathbb{N}$ and $f \in C_{0}^{k}(\mathbb{R})$. Then, for any $n \in \mathbb{N}$ and for any $i \leq k, \int_{\mathbb{R}} f(x) \delta_{n}^{(i)}(x) d x$ exists and

$$
\lim _{n \rightarrow \infty} \int_{\mathbb{R}} f(x) \delta_{n}^{(i)}(x) d x=(-1)^{i} f^{(i)}(0)\left(=u^{(i)}(f)\right) .
$$

Proof. Since both $f$ and $\eta^{(i)}$ have a modulus of uniform continuity, we can integrate $f(x) \delta_{n}^{(i)}(x)$ within $\mathrm{RCA}_{0}$. Thus, we can prove this theorem in the usual way within $\mathrm{RCA}_{0}$.

Now, we apply the Dirac delta function to a larger class of functions (e.g. continuous or $\mathrm{C}^{k}$ ). By [22], we know that Fourier analysis can be developed in $\mathrm{RCA}_{0}$ or $W W K L_{0}$, depending on the underlying class of functions. As the Dirac delta function is an important tool in Fourier analysis, we can expect the properties of this function to be provable in $\mathrm{WWKL}_{0}$.

12. Theorem. The following assertions are pairwise equivalent over $\mathrm{RCA}_{0}$.

1. WWKL $L_{0}$.

2. For any bounded support continuous function $f$ on $\mathbb{R}$, and large enough $n$, the integral $\int_{\mathbb{R}} f(x) \delta_{n}(x) d x$ exists and

$$
\lim _{n \rightarrow \infty} \int_{\mathbb{R}} f(x) \delta_{n}(x) d x=f(0)(=u(f)) .
$$

3. For any bounded support $\mathcal{C}^{k}$ function $f$ on $\mathbb{R}$, for large enough $n$, and for any $i \leq k$, the integral $\int_{\mathbb{R}} f(x) \delta_{n}^{(i)}(x) d x$ exists and

$$
\lim _{n \rightarrow \infty} \int_{\mathbb{R}} f(x) \delta_{n}^{(i)}(x) d x=(-1)^{i} f^{(i)}(0)\left(=u^{(i)}(f)\right) .
$$

Proof. We first show $1 \rightarrow 3$. We argue in $\mathrm{WWKL}_{0}$. Let $f$ be a $\mathcal{C}^{k}$ function. By the definition of $\mathrm{C}^{k}$ functions, we can find $K \in \mathbb{N}$ such that $\sup \left\{\left|f^{(i)}(x)-f^{(i)}(0)\right| \mid x \in\left[-2^{-K}, 2^{-K}\right], i \leq k\right\}<1$. Indeed, we can find such $K \in \mathbb{N}$ since $\bigcap_{i \leq k}\left(f^{(i)}\right)^{-1}\left(B\left(f^{(i)}(0) ; 1\right)\right)$ is a non-empty open set. By Theorem 10. for any $n \geq K$ and for any $i \leq k, \int_{\mathbb{R}} f(x) \delta_{n}^{(i)}(x) d x$ exists. Then, we can prove $\lim _{n \rightarrow \infty} \int_{\mathbb{R}} f(x) \delta_{n}^{(i)}(x) d x=(-1)^{i} f^{(i)}(0)$ as usual.

The implication $3 \rightarrow 2$ is trivial, so we show $\neg 1 \rightarrow \neg 2$. We argue in RCA 0 , assuming $\neg$ WWKL. Consider the bounded continuous function $f:[0,1] \rightarrow \mathbb{R}$ constructed in $(3)$ in the proof of Theorem 10 . Note that $f$ is not Riemann integrable and that $f(0)=f(1)=0$. Define the continuous function $g: \mathbb{R} \rightarrow \mathbb{R}$ with bounded support as

$$
g(x)= \begin{cases}0 & \text { if } x \leq 0 \text { or } x \geq 1 \\ 2^{-m} f(t) & \text { if } x=2^{-m-1}+2^{-m-1} t \text { for some } m \in \mathbb{N} \text { and } t \in[0,1] .\end{cases}
$$

Now, assume that for some $n \in \mathbb{N}, \int_{\mathbb{R}} g(x) \delta_{n}(x) d x$ exists. Then, for some $m \in \mathbb{N},\left[2^{-m-1}, 2^{-m}\right] \subseteq$ $\left[0,2^{-n-1}\right]$. Since $\delta_{n}(x)>0$ on $\left[0,2^{-n-1}\right]$, the integral $\int_{2^{-m-1}}^{2^{-m}} g(x) d x$ exists. This implies that $\int_{0}^{1} f(x) d x$ exists, which contradicts the definition of $f$. This completes the proof of $\neg 1 \rightarrow \neg 2$.

13. Remark. Defining $H_{n}(x)=\int_{-\infty}^{x} \delta_{n}(t) d t$, the limit of $\left\{H_{n}\right\}_{n \in \mathbb{N}}$ (as a distribution) can be considered as the Heaviside function $H(x)$. Within $\mathrm{RCA}_{0}$, we can prove that the derivative of the Heaviside function (as a distribution) is the Dirac delta function for $C_{0}^{k}(\mathbb{R})$-functions. However, for a bounded support continuous (or $\mathrm{C}^{k}$ ) function $f$, we need $\mathrm{WKL}_{0}$ to show the existence of $\int_{\mathbb{R}} f(x) H_{n}(x) d x$. Thus, we need $\mathrm{WKL}_{0}$ to handle the Heaviside function. Moreover, we can show 
that a version of the distributional property $\frac{d H}{d x}=\delta(x)$ of the Heaviside function for $C^{k}$-functions is equivalent to $\mathrm{WKL}_{0}$.

14. Remark. In nonstandard second-order arithmetic, we can consider the Dirac delta function as $\delta_{\omega}(x)$ for a nonstandard number $\omega$. The nonstandard versions of items 2 and 3 of Theorem 12 can be formalized within a system of nonstandard second-order arithmetic ns-WWKL $\mathrm{L}_{0}$ (for ns-WWKL $\mathrm{W}_{0}$, see 17]). It is still open whether the nonstandard versions of items 2 and 3 are equivalent to ns-WWKL $L_{0}$. In the setting of ERNA's Reverse Mathematics, the nonstandard version of item (2) is equivalent to $\Pi_{1}$-TRANS. This is the topic of the next section.

\section{The Dirac Delta in ERNA's Reverse Mathematics}

In this section, we consider the Dirac Delta function in the context of ERNA's Reverse Mathematics. For details concerning the latter, we refer the reader to [14]. For an introduction to ERNA, we refer to 9 20. In two words, ERNA (or Elementary Recursive Nonstandard Analysis) is a system of Nonstandard Analysis based on the weak system $I \Delta_{0}+$ exp. The 'ER' in ERNA refers to the fact that new functions may be defined in ERNA using primitive recursion (e.g. summation, yielding the Riemann integral) as long as the resulting function is bounded by a fixed iteration of the exponential function. In this way, most functions of calculus are available in ERNA. For instance, in [21. Section 13], Suppes and Chuaqui derive the equation of the catenary, determine the flux in a blood vessel, study the Poisson process, and consider the Fresnel integral for diffraction phenomena, all in (a predecessor of) ERNA. In [2], a good deal of calculus is developed in the same system, including the first fundamental theorem of integration. As discussed above for Peano's theorem, all results hold 'up to infinitesimals'.

Here, we will prove that ERNA's version of the Dirac Delta Theorem is equivalent to $\Pi_{1}$-TRANS. First, we recall the latter transfer principle and some of its essential features. Then, we develop some necessary analysis. Finally, we prove the equivalence between the Dirac Delta Theorem (or DDT) and $\Pi_{1}$-TRANS. Unless explicitly stated otherwise, theorems are proved in ERNA.

3.1. Universal Transfer. In this paragraph, we recall the transfer principle for universal formulas and its properties (see $[9,14$ ). This principle expresses Leibniz' law that the 'same laws' should hold for standard and nonstandard numbers alike.

Let $L^{s t}$ be ERNA's language $L$ without the symbols $\omega, \varepsilon, \approx$ and min. Note that standard parameters are allowed in the formula $\varphi(x)$ in the following transfer principle.

15. Principle ( $\Pi_{1}$-TRANS). Let $\varphi(x) \in L^{\text {st }}$ be quantifier-free. Then

$$
\left(\forall^{s t} x\right) \varphi(x) \rightarrow(\forall x) \varphi(x) .
$$

Obviously, the scope of this principle (also called 'universal transfer' or ' $\Pi_{1}$-transfer') is quite limited. Indeed, $\Pi_{1}$-transfer is limited to formulas of $L^{s t}$. Hence, a formula cannot be transferred if it contains, for instance, ERNA's exponential function $\sum_{n=0}^{\omega} \frac{x^{n}}{n !}$ or similar objects not definable in $L^{s t}$. This is quite a limitation, especially for the development of basic analysis. In [14, the scope of $\Pi_{1}$-transfer was expanded so as to be applicable to objects like ERNA's exponential. We briefly sketch these results here.

First, we label some terms which, though not part of $L^{s t}$, are 'nearly as good' as standard for the purpose of transfer. As in [9, Notation 57], the variable $\omega^{\prime}$ in $\left(\forall \omega^{\prime}\right)$ runs over the infinite 
hypernaturals. As in 14, we assume that no term involves ERNA's minimum ${ }^{2}$ operator, unless explicitly stated otherwise.

16. Definition. Let the term $\tau(n, \vec{x})$ be standard, i.e. not involve $\omega$ or $\approx$. We say that $\tau(\omega, \vec{x})$ is near-standard if ERNA proves

$$
(\forall \vec{x})\left(\forall \omega^{\prime}\right)\left(\tau(\omega, \vec{x}) \approx \tau\left(\omega^{\prime}, \vec{x}\right)\right) .
$$

An atomic inequality $\tau(\omega, \vec{x}) \leq \sigma(\omega, \vec{x})$ is called near-standard if both members are. Since $x=y$ is equivalent to $x \leq y \wedge x \geq y$, and $\mathcal{N}(x)$ to $\lceil x\rceil=|x|$, any internal formula $\varphi(\omega, \vec{x})$ can be assumed to consist entirely of atomic inequalities; it is called near-standard if it is made up of near-standard atomic inequalities.

We list several examples of near-standard terms and formulas.

17. Example. Consider $\sqrt{x}$ which satisfies $(\sqrt{x})^{2} \approx x$ for $x \in[0,1]$. This 'square root function' is defined as $\sqrt{x}:=(\mu n \leq \omega)\left[\left(\frac{n}{\omega}\right)^{2} \geq x\right] / \omega$ (see also 8, Example 5.2.4] and 9, Theorem 58]). The following formula is near-standard:

$$
0<x<y^{2}<1 \rightarrow \sqrt{x}<y .
$$

It is worth mentioning that there is no number $q$ in ERNA $+\Pi_{1}$-TRANS such that $q^{2}=2$. Thus, no computational process can ever produce $\sqrt{2}$, not even in principle. Similarly, most results in Theorem 2 only yield solutions with an infinitesimal error. This suggests that ERNA corresponds better to 'computation in the real world' than other frameworks, as suggested by Sommer and Suppes in 20]. For the following example, we need some notation.

18. Notation. We write $a \ll b$ for $a \leq b \wedge a \not b b$ and $a \lesssim b$ for $a \leq b \vee a \approx b$.

19. Example. Consider $\arctan x:=\int_{0}^{\underline{x}} \frac{1}{1+t^{2}} d_{\rho} t$, where $\rho$ is an infinitely fine partition (see Definitions 2728 and 29 below). Defining $\pi:=4 \sum_{k=0}^{\omega} \frac{(-1)^{k}}{2 k+1}$, the following formula is near-standard:

$$
x>0 \rightarrow \arctan x \leq \frac{\pi}{2} .
$$

In stronger theories of Nonstandard Analysis, near-standard terms such as $\arctan x$ and $\sqrt{x}$ would be converted to standard terms by the 'standard part map' st $(x)$ which satisfies $\operatorname{st}(x+\varepsilon)=x$, for $\varepsilon \approx 0$ and standard $x$. However, ERNA does not have such a map and hence functions of basic analysis like $\arctan x$ and $\sqrt{x}$, defined above, are not allowed in $\Pi_{1}$-TRANS. Moreover, full transfer for near-standard formulas is actually impossible: formulas (6) and (7) are true for all rational $x, y$, but there are hyperrational counterexamples. Nonetheless, the following formulas hold for all $x, y$

$$
x>0 \rightarrow \arctan x \lesssim \frac{\pi}{2} \text { and } 0<x<y^{2}<1 \rightarrow \sqrt{x} \lesssim y .
$$

Replacing ' $\leq$ ' with ' $\approx$ ' for near-standard formulas is the key idea behind generalizing the scope of transfer, as is clear from Definition 20 and 80 below.

20. Definition. Given a near-standard formula $\varphi(\vec{x})$, let $\bar{\varphi}(\vec{x})$ be the formula obtained by replacing every positive (negative) occurrence of a near-standard inequality $\leq$ with $\lesssim(\ll)$.

Now consider the following principle, called 'bar transfer'.

\footnotetext{
${ }^{2}$ The minimum operator allows us to convert certain existential formulas into quantifier-free ones. Thus, without the imposed limitation, not all internal quantifier-free formulas would be decidable as proved in 9 . Theorem 50].
} 
21. Principle $\left(\bar{\Pi}_{1}\right.$-TRANS). Let $\varphi(x)$ be near-standard and quantifier-free. Then

$$
\left(\forall^{s t} x\right) \varphi(x) \rightarrow(\forall x) \bar{\varphi}(x) .
$$

As for $\Pi_{1}$-transfer, standard parameters are allowed in the previous transfer principle. Despite its much wider scope, bar transfer is equivalent to $\Pi_{1}$-transfer.

22. Theorem. In ERNA, the schemas $\Pi_{1}$-TRANS and $\bar{\Pi}_{1}$-TRANS are equivalent.

Proof. For special $\Pi_{1}$-formulas, this was done in $[10, \S 3]$ with a relatively easy proof. For general $\Pi_{1}$-formulas, the proof becomes significantly more involved (see 14 . Theorem 9]). Ironically, we have to resort to $\varepsilon-\delta$ techniques.

The following theorem guarantees that near-standard terms are automatically finite for finite arguments. This is surprising, since Definition 16 does not mention the (in)finitude of near-standard terms. Thus, near-standardness seems to be a natural property.

23. Theorem. A near-standard term $\tau(\vec{x}, \omega)$ is finite for finite $\vec{x}$.

Proof. To improve readability, we assume that $\vec{x}$ is just $n$. The following sentence expresses that $\tau(n, \omega)$ is near-standard: $(\forall n)\left(\forall \omega^{\prime}\right)\left(\tau\left(n, \omega^{\prime}\right) \approx \tau(n, \omega)\right)$. This implies

$$
\left(\forall^{s t} k\right)(\forall n)\left(\forall \omega^{\prime}\right)\left(\left|\tau\left(n, \omega^{\prime}\right)-\tau(n, \omega)\right|<1 / k\right),
$$

and also

$$
\left(\forall^{s t} k\right)\left(\forall \omega^{\prime}\right)\left(\forall n \leq \omega_{1}\right)\left(\left|\tau\left(n, \omega^{\prime}\right)-\tau(n, \omega)\right|<1 / k\right),
$$

where $\omega_{1}$ is a fixed infinite hypernatural number. By underflow (see 9 , Theorem 54]), we have

$$
\left(\forall^{s t} k\right)\left(\exists^{s t} M\right)(\forall m \geq M)\left(\forall n \leq \omega_{1}\right)(|\tau(n, m)-\tau(n, \omega)|<1 / k) .
$$

Apply this formula for $k=1$ to obtain finite $M_{1}$ such that $\left(\forall^{s t} n\right)\left(\left|\tau\left(n, M_{1}\right)-\tau(n, \omega)\right|<1\right.$. By [9. Theorem 27], the term $\tau\left(n, M_{1}\right)$ is finite for finite $n$ and we are finished.

The Big five systems of Reverse Mathematics are part of second-order arithmetic. Thus, objects of ordinary mathematics, like functions from $\mathbb{R}$ to $\mathbb{R}$, are coded into sets of natural numbers. In ERNA, we take a different approach: We observe that most objects of ordinary mathematics can be approximated to within infinitesimal error by ERNA's near-standard terms. As shown in this section, near-standard terms also have good logical properties.

3.2. Continuity and integration. In this paragraph, we sketch the development of Riemann integration and continuity inside ERNA. First, we introduce two notions of continuity in ERNA. We always assume $a$ and $b$ to be finite.

24. Definition. A function $f(x)$ is 'continuous over $[a, b]$ ' if

$$
(\forall x, y \in[a, b])(x \approx y \rightarrow f(x) \approx f(y)) .
$$

A function $f(x)$ is called 'S-continuous over $[a, b]$ ' if

$$
\left(\forall^{s t} k\right)\left(\exists^{s t} N\right)\left(\forall^{s t} x, y \in[a, b]\right)(|x-y|<1 / N \rightarrow|f(x)-f(y)|<1 / k) .
$$

The attentive reader has noted that $(9)$ and $(10)$ pertain to uniform continuity. We believe this to be justified for the following reason. If we limit the variable $x$ in 9 to $\mathbb{Q}$, the function $\frac{1}{x^{2}-2}$ satisfies the resulting formula, although this function is infinite in the interval $[-2,2]$. Similarly, the function $g(x)$, defined as 1 if $x^{2}<2$ and 0 if $x^{2} \geq 2$, satisfies (9) with $x$ limited to $\mathbb{Q}$, but this 
function has a jump in its graph. The same holds for pointwise $\varepsilon-\delta$ continuity. Thus, both are not suitable for our purposes. Note that a similar situation occurs in constructive analysis.

Now consider the following 'continuity principle'.

25. Principle. A near-standard function is continuous over $[a, b]$ if it is $S$-continuous over $[a, b]$.

In the mathematical practice of Physics, an intuitive 'calculus with infinitesimals' is used, in favour of Weierstrass' $\varepsilon-\delta$ analysis. In particular, the notion of continuity expressed by $(9)$ is used, rather than 10. Thus, one could argue that Physicists implicitly make use of the continuity principle, and hence of $\Pi_{1}$-TRANS, by the following theorem.

26. Theorem. In ERNA, the continuity principle is equivalent to $\Pi_{1}$-TRANS.

Proof. See [14, Theorem 43].

Next, we define Riemann integration inside ERNA.

27. Definition. A partition $\pi$ of $[a, b]$ is a vector $\left(x_{1}, \ldots, x_{n+1}, t_{1}, \ldots t_{n}\right)$ such that $x_{i} \leq t_{i} \leq x_{i+1}$ for all $1 \leq i \leq n$ and $a=x_{1}$ and $b=x_{n+1}$. The 'mesh' of $\pi$ is $|\pi|:=\max _{1 \leq i \leq n}\left(x_{i+1}-x_{i}\right)$. The partition $\pi$ is 'infinitely fine' if $|\pi| \approx 0$.

By the remark after [14, Theorem 17], we know that ERNA can code partitions into hypernaturals and vice versa. Thus, the following definition is inside ERNA.

28. Definition (Riemann Integration). Let $f$ be a function defined on $[a, b]$.

1. The Riemann sum $S_{\pi}(f)$ corresponding to a partition $\pi=\left(x_{1}, \ldots, x_{n+1}, t_{1}, \ldots, t_{n}\right)$ is defined as the sum $\sum_{i=1}^{n} f\left(t_{i}\right)\left(x_{i+1}-x_{i}\right)$.

2. The function $f$ is called 'Riemann integrable on $[a, b]$ ' if all Riemann sums of infinitely fine partitions are finite and infinitely close to each other. If so, the Riemann sum corresponding to the infinitely fine partition $\pi$ of $[a, b]$ is denoted by $\int_{a}^{b} f(x) d_{\pi} x$.

3. A function $f$ is called 'absolutely integrable over $[a, b]$ ' if $|f|$ is Riemann integrable there.

4. A function is called 'everywhere integrable' if it is Riemann integrable over the interval $[-\omega, \omega]$ for all infinite $\omega$ and all integrals are infinitely close.

If $\pi$ is a partition of $[a, b]$ and $a<c<d<b$, we sometimes write $\int_{c}^{d} f(x) d_{\pi} x$ instead of $\int_{c}^{d} f(x) d_{\rho} x$, where $\rho$ is $\pi$ limited to $[c, d]$.

29. Definition. If $\pi$ is an infinitely fine partition of $[a, b]$, we denote by $\underline{x}$ the least partition point not exceeding $x$. If $f$ is integrable over $[a, b]$, we define

$$
F_{\pi}(x):=\int_{a}^{\underline{x}} f(t) d_{\pi} t .
$$

For completeness, we recall ERNA's weight function $\|x\|$ which is defined as $\| \pm n / m\|=\max (n, m)$, for hypernatural $n, m$ with $m \neq 0$ (see [9, Theorem 23]).

By 14, Theorem 50], we know that the statement a near-standard function is integrable over $[a, b]$ if it is $S$-continuous there is equivalent to $\Pi_{1}$-TRANS. Now consider the following, seemingly weaker principle.

30. Principle. A near-standard function which is S-continuous and bounded over [0,1], is integrable there. 
Although this principle seems weaker than the Riemann integrability principle, we have the following theorem.

31. Theorem. In ERNA, Principle 30 is equivalent to $\Pi_{1}$-TRANS.

Proof. The reverse implication is immediate from Theorems 16 and 36 in [14. Conversely, assume that Principle 30 holds and consider a quantifier-free formula $\varphi$ of $L^{s t}$ such that $\varphi(n)$ is valid for all $n \in \mathbb{N}$. Let $f$ be near-standard, S-continuous and bounded over $[0,1]$, with Riemann integral infinitely close to 1 . By cases, we define the near-standard function

$$
g(x)=\left\{\begin{array}{ll}
f(x) & (\forall n \leq\|x\|) \varphi(n) \\
x & \text { otherwise }
\end{array} .\right.
$$

It is clear that $g(x)$ is bounded on $[0,1]$. As $\varphi(n)$ is true for all standard $n$, we have $g(x)=f(x)$ for all standard $x$, by definition. Hence $g(x)$ is also S-continuous over $[0,1]$. In particular, Principle 30 applies to $g$. Overflow (see 9. Theorem 54]) applied to $\left(\forall^{s t} n\right) \varphi(n)$ yields $\left(\forall n \leq \omega_{1}\right) \varphi(n)$ and hence $g(x)=f(x)$ for all $x$ such that $\|x\| \leq \omega_{1}$. Then put $\omega_{2}=\left\lfloor\omega_{1} / 2\right\rfloor$ and consider the equidistant partition with mesh $1 / \omega_{2}$ and points $t_{i}=\frac{x_{i+1}+x_{i}}{2}$. As $\left\|t_{i}\right\| \leq \omega_{1}$, it is clear that $g\left(t_{i}\right)=f\left(t_{i}\right)$ for $1 \leq i \leq \omega_{2}$ and hence the Riemann sum of $g$ corresponding to this partition is infinitely close to 1 .

Now suppose there is a (necessarily infinite) hypernatural $n_{1}$ such that $\neg \varphi\left(n_{1}\right)$. As Euclid's proof of the infinitude of the prime numbers can easily be formalised in ERNA, there is an $n_{0} \geq n_{1}$ which is prime. By the definition of $g(x)$, there follows $g(x)=x$ if $\|x\| \geq n_{1}$. Then consider the equidistant partition with mesh $1 / n_{0}$ and points $t_{i}=\frac{x_{i+1}+x_{i}}{2}$. The corresponding Riemann sum is easily calculated and has value $\frac{1}{2}$. By Principle 30 there holds $1 \approx \frac{1}{2}$. Obviously, this is impossible and the assumption that there is a number $n_{1}$ such that $\neg \varphi\left(n_{1}\right)$ is false. Hence, we have $\varphi(n)$ for all hypernatural $n$. This implies $\Pi_{1}$-TRANS and we are done.

In classical Reverse Mathematics, the statement a function is integrable over $[a, b]$ if it is continuous over $[a, b]$ is equivalent to WKL. Restricting to bounded functions results in a statement that is equivalent to WWKL (see Theorem 10, and the latter is strictly weaker than WKL. By the previous theorem, it seems that in ERNA's Reverse Mathematics, the principles WKL and WWKL coincide. This explains why, in classical Reverse Mathematics, DDT is equivalent to WWKL, whereas in ERNA's Reverse Mathematics, DDT is equivalent to $\Pi_{1}$-TRANS (see Theorem 38).

3.3. Dirac delta function. In this paragraph, we introduce the Dirac delta function and prove that ERNA's version of the Dirac Delta Theorem is equivalent to $\Pi_{1}$-TRANS.

32. Definition (Delta function). Let $f(x)$ be absolutely integrable everywhere with integral infinitely close to one. For nonzero $\varepsilon \approx 0, \delta_{\varepsilon}(x):=f(x / \varepsilon) / \varepsilon$ is called a 'delta function'.

Note that, with this definition, $\delta_{\varepsilon}(x)$ is indeed a function. Theorem 35 below shows that delta functions exhibit the typical 'infinite at zero and zero everywhere else' behaviour. The following definition and theorem are concerned with the integration of delta functions.

33. Definition. A function is called ' $\varepsilon$-integrable over $[a, b]$ ' if there is a finite $q$ such that for all hyperfine partitions $\pi$ of $[a, b]$ such that $\frac{|\pi|}{\varepsilon} \approx 0$, we have $S_{\pi}(f) \approx q$.

The condition $\frac{|\pi|}{\varepsilon} \approx 0$ in the definition guarantees that a hyperfine partition is fine enough to allow rescaling by $\varepsilon$ without affecting its 'hyperfineness'. This is clear from the following theorem. 
34. Theorem. Let $f$ be integrable over $[a / \varepsilon, b / \varepsilon]$ with $0<\varepsilon \approx 0$. Then $f(x / \varepsilon) / \varepsilon$ is $\varepsilon$-integrable over $[a, b]$ and we have

$$
\int_{a}^{b} f\left(\frac{x}{\varepsilon}\right) \frac{1}{\varepsilon} d_{\pi} x \approx \int_{a / \varepsilon}^{b / \varepsilon} f(x) d_{\rho} x,
$$

for any hyperfine partition $\pi$ of $[a, b]$ such that $\frac{|\pi|}{\varepsilon} \approx 0$.

Proof. Let $\pi=\left(x_{1}, \ldots, x_{k+1}, t_{1}, \ldots, t_{k}\right), a, b$ and $f$ be as in the theorem. Then $S_{\pi}(f)=$ $\sum_{i=1}^{k} f\left(t_{i} / \varepsilon\right) / \varepsilon\left(x_{i+1}-x_{i}\right)$ and define $y_{i}=x_{i} / \varepsilon$ and $s_{i}=t_{i} / \varepsilon$. Since $\frac{|\pi|}{\varepsilon} \approx 0$, the list $\pi^{\prime}=$ $\left(y_{1}, \ldots, y_{k+1}, s_{1}, \ldots, s_{k}\right)$ is a hyperfine partition of $[a / \varepsilon, b / \varepsilon]$. As $f$ is integrable over the latter interval, we have

$$
S_{\pi}(f)=\sum_{i=1}^{k} f\left(t_{i} / \varepsilon\right) / \varepsilon\left(x_{i+1}-x_{i}\right)=\sum_{i=1}^{k} f\left(s_{i}\right)\left(y_{i+1}-y_{i}\right) \approx \int_{a / \varepsilon}^{b / \varepsilon} f(x) d_{\rho} x,
$$

where $\rho$ is a hyperfine partition of $[a / \varepsilon, b / \varepsilon]$. This finishes the proof.

35. Theorem. The delta function $\delta_{\varepsilon}(x)$ is $\varepsilon$-integrable. Moreover, for all $a<-\varepsilon^{\prime}<\varepsilon^{\prime}<b$ with $\varepsilon / \varepsilon^{\prime} \approx 0$, we have $\int_{a}^{b} \delta_{\varepsilon}(x) d_{\pi} x \approx \int_{-\varepsilon^{\prime}}^{\varepsilon^{\prime}} \delta_{\varepsilon}(x) d_{\pi} x \approx 1$.

Proof. Let $\delta_{\varepsilon}(x)=f(x / \varepsilon) / \varepsilon$ be a delta function and fix $0<\varepsilon^{\prime} \approx 0$ such that $\varepsilon^{\prime} / \varepsilon$ is infinite. Let $a, b$ be as in the theorem and let $\rho$ be a partition of $[a / \varepsilon, b / \varepsilon]$. As $f(x)$ is integrable everywhere, we have $\int_{a / \varepsilon}^{b / \varepsilon} f(x) d_{\rho} x \approx \int_{-\varepsilon^{\prime} / \varepsilon}^{\varepsilon^{\prime} / \varepsilon} f(x) d_{\rho} x \approx 1$. Let $\pi$ be a partition of $[a, b]$ such that $\frac{|\pi|}{\varepsilon} \approx 0$. By the previous theorem, we have $\int_{a / \varepsilon}^{b / \varepsilon} f(x) d_{\rho} x \approx \int_{a}^{b} f\left(\frac{x}{\varepsilon}\right) \frac{1}{\varepsilon} d_{\pi} x$ and $\int_{-\varepsilon^{\prime} / \varepsilon}^{\varepsilon^{\prime} / \varepsilon} f(x) d_{\rho} x \approx \int_{-\varepsilon^{\prime}}^{\varepsilon^{\prime}} f\left(\frac{x}{\varepsilon}\right) \frac{1}{\varepsilon} d_{\pi} x$.

Thus, we see that $\delta_{\varepsilon}(x)$ must be infinitesimal almost everywhere outside of an infinitely small region around zero, where it is infinite. Now consider the following principle, called 'Dirac Delta Theorem', or 'DDT' for short. It shows that delta functions select the value $f(0)$ of a function $f$.

36. Principle (DDT). Let $g$ be near-standard and $S$-continuous on $[a, b]$ and let $\pi$ be a hyperfine partition of $[a, b]$. If $\frac{|\pi|}{\varepsilon} \approx 0$ and $a \ll 0 \ll b$ and $\delta_{\varepsilon}(x)$ is a delta function, then

$$
\int_{a}^{b} g(x) \delta_{\varepsilon}(x) d_{\pi} x \approx g(0)
$$

37. Theorem. The Dirac Delta Theorem is provable in ERNA $+\Pi_{1}$-TRANS.

Proof. Let $g$ and $\pi$ be as in the theorem. By Theorem 35 there is an $\varepsilon^{\prime} \approx 0$ such that $\int_{a}^{b} \delta_{\varepsilon}(x) d_{\pi}(x) \approx$ $\int_{-\varepsilon^{\prime}}^{\varepsilon^{\prime}} \delta_{\varepsilon}(x) d_{\pi} x \approx 1$. This implies that $\int_{a}^{-\varepsilon^{\prime}} \delta_{\varepsilon}(x) d_{\pi}(x) \approx 0$, which in turn yields

$$
\int_{a}^{-\varepsilon^{\prime}} g(x) \delta_{\varepsilon}(x) d_{\pi}(x) \lesssim M_{1} \int_{a}^{-\varepsilon^{\prime}} \delta_{\varepsilon}(x) d_{\pi}(x) \approx 0,
$$

where $M_{1}$ is the finite maximum of $g$ on $\left[a,-\varepsilon^{\prime}\right]$, provided by ERNA's Weierstrass Extremum Theorem (see 14, Theorem 12]). Similarly, we prove $\int_{\varepsilon^{\prime}}^{b} g(x) \delta_{\varepsilon}(x) d_{\pi}(x) \approx 0$.

Now let $t_{j_{1}}\left(t_{j_{2}}\right)$ be the least (largest) point in the partition $\pi$ such that $t_{j_{1}} \geq \varepsilon^{\prime}\left(t_{j_{2}} \leq-\varepsilon^{\prime}\right)$. Define the finite numbers $M$ and $m$ as $\max _{j_{2} \leq i \leq j_{1}} g\left(t_{i}\right)$ and least $j_{2} \leq i \leq j_{1} g\left(t_{i}\right)$, respectively. By [9. §5.1], these terms are available in ERNA. This yields

$$
\int_{a}^{b} g(x) \delta_{\varepsilon}(x) d_{\pi} x \approx \int_{-\varepsilon^{\prime}}^{\varepsilon^{\prime}} g(x) \delta_{\varepsilon}(x) d_{\pi} x \lesssim M \int_{-\varepsilon^{\prime}}^{\varepsilon^{\prime}} \delta_{\varepsilon}(x) d_{\pi} x \approx M .
$$

Similarly, one proves $m \lesssim \int_{a}^{b} g(x) \delta_{\varepsilon}(x) d_{\pi} x$. By the continuity principle, $g$ is continuous, implying $m \approx g(0) \approx M$, and the theorem follows. 
Note that we may replace $a$ and $b$ with infinite numbers, if we assume that $f$ is zero outside an interval with finite extremes or is integrable everywhere. In 14 , conditions similar to $\frac{|\pi|}{\varepsilon} \approx$ 0 appear in ERNA's version of the Fundamental Theorem of Calculus and Peano's Theorem. Actually, these conditions hide $\varepsilon-\delta$ statements in an elegant way. Intuitively, they express that $|\pi|$ is infinitesimal, even compared to $\varepsilon$. In [13, a framework is presented that removes these conditions by incorporating different degrees of magnitude directly into the theory ERNA.

38. Theorem. In ERNA, DDT is equivalent to $\Pi_{1}$-TRANS.

Proof. The reverse implication is immediate from Theorem 37. Conversely, assume that DDT holds and consider a quantifier-free formula $\varphi$ of $L^{s t}$ such that $\varphi(n)$ is true for all $n \in \mathbb{N}$. Let $g$ be near-standard and S-continuous over $[a, b]$. By cases, we define the near-standard function

$$
h(x)=\left\{\begin{array}{ll}
g(x) & (\forall n \leq\|x\|) \varphi(n) \\
\|x\| & \text { otherwise }
\end{array} .\right.
$$

As $\varphi(n)$ is true for all $n \in \mathbb{N}$, we have $h(x)=g(x)$ for all standard $x$ and hence $h(x)$ is also S-continuous. In particular, DDT applies to $h$.

Now suppose there is a (necessarily infinite) hypernatural $n_{1}$ such that $\neg \varphi\left(n_{1}\right)$ and let $n_{0} \geq n_{1}$ be prime and large enough such that $\frac{1}{n_{0} \varepsilon} \approx 0$. By the definition of $h(x)$, we have $h(x)=\|x\|$ if $\|x\| \geq n_{0}$. Then consider the equidistant partition $\pi$ of $[a, b]$ with mesh $1 / n_{0}$ and points $t_{i}=\frac{x_{i+1}+x_{i}}{2}$ for $1 \leq i \leq m_{0}$. Note that $\frac{|\pi|}{\varepsilon} \approx 0$. The corresponding Riemann sum is easily calculated:

$$
\begin{aligned}
\int_{a}^{b} h(x) \delta_{\varepsilon}(x) d_{\pi} x=\sum_{i=1}^{m_{0}} h\left(t_{i}\right) \delta_{\varepsilon}\left(t_{i}\right)\left(x_{i}-x_{i-1}\right) & =\sum_{i=1}^{m_{0}}\left\|t_{i}\right\| \delta_{\varepsilon}\left(t_{i}\right)\left(x_{i}-x_{i-1}\right) \\
& \geq 2 n_{0} \sum_{i=1}^{m_{0}} \delta_{\varepsilon}\left(t_{i}\right)\left(x_{i}-x_{i-1}\right) \\
& =2 n_{0} \int_{a}^{b} \delta_{\varepsilon}(x) d_{\pi} x \gg n_{0}
\end{aligned}
$$

By DDT, we have $\int_{a}^{b} h(x) \delta_{\varepsilon}(x) d_{\pi} x \approx h(0)$, but, by definition, $h(0)=g(0)$. By Theorem $23, g(0)$ is a finite number, whereas, by [14, the integral $\int_{a}^{b} h(x) \delta_{\varepsilon}(x) d_{\pi} x$ is infinite. This is a contradiction and hence, the assumption that there is a number $n_{1}$ such that $\neg \varphi\left(n_{1}\right)$ is false. Thus, we have $\varphi(n)$ for all $n$. This implies $\Pi_{1}$-TRANS and we are done.

3.4. Heaviside function. In this paragraph, we introduce the Heaviside function and prove ERNA's version of the essential property $\int \frac{d H}{d x}(x) \cdot f(x) d x=f(0)$. Thus, we observe that the derivative of the Heaviside function is a delta function. We also show that this property is equivalent to $\Pi_{1}$-TRANS.

First, we introduce the notions of differentiability and derivative in ERNA. For brevity, we sometimes write ' $\Delta_{h} f(x)$ ' instead of $\frac{f(x+h)-f(x)}{h}$.

39. Definition. A function is called 'differentiable over $(a, b)$ ' if

$$
\begin{aligned}
& \left(\forall^{s t} k\right)\left(\exists^{s t} N\right)\left(\forall^{s t} h, h^{\prime}\right)\left(\forall^{s t} x \in(a, b)\right) \\
& \quad\left[x+h \in[a, b] \wedge x+h^{\prime} \in[a, b] \wedge 0<|h|,\left|h^{\prime}\right|<\frac{1}{N} \rightarrow\left|\Delta_{h} f(x)-\Delta_{h^{\prime}} f(x)\right|<\frac{1}{k}\right] .
\end{aligned}
$$

For $\varepsilon \approx 0$, the function $\Delta_{\varepsilon} f(x)$ is the called 'the derivative of $f$ '. By the following theorem, proved in ERNA $+\Pi_{1}$-TRANS, the derivative is uniquely defined, up to infinitesimals. Thus, 
$f^{\prime}(x)$ is called 'the derivative of $f(x)$ '. Like in the case of continuity, we use the uniform notion of differentiability. Thus, it is not surprising that the derivative is also continuous.

40. Theorem. A near-standard function, differentiable over $(a, b)$, satisfies $\Delta_{\varepsilon} f(x) \approx \Delta_{\varepsilon^{\prime}} f(x)$, for all $a \ll x \ll b$ and $\varepsilon, \varepsilon^{\prime} \approx 0$. Moreover, for all $\varepsilon \approx 0, \Delta_{\varepsilon} f(x)$ is continuous for $a \ll x \ll b$.

Proof. By Theorem 22, we may use bar transfer. In (15), fix finite $k=k_{0}+1$ and find a finite number $N_{0}$ such that the innermost universal formula holds. Applying bar transfer to the resulting formula yields, for all $h, h^{\prime}$ and all $x \in(a, b)$, that

$$
x+h \in[a, b] \wedge x+h^{\prime} \in[a, b] \wedge 0<|h|,\left|h^{\prime}\right|<\frac{1}{N_{0}} \rightarrow\left|\Delta_{h} f(x)-\Delta_{h^{\prime}} f(x)\right| \lesssim \frac{1}{k_{0}+1}
$$

Thus, we see that for $h, h^{\prime} \approx 0$ and $a \ll x \ll b$, we have $\left|\Delta_{h} f(x)-\Delta_{h^{\prime}} f(x)\right|<\frac{1}{k_{0}}$. The latter formula is true for arbitrary $k_{0}$ and we have $\Delta_{h} f(x) \approx \Delta_{h^{\prime}} f(x)$ if $h, h^{\prime} \approx 0$.

Now choose two points $x \approx y$ such that $a \ll x<y \ll b$. If $y-x=\varepsilon$, then

$$
\Delta_{\varepsilon} f(x)=\frac{f(x+\varepsilon)-f(x)}{\varepsilon}=\frac{f(y)-f(y-\varepsilon)}{\varepsilon}=\frac{f(y-\varepsilon)-f(y)}{-\varepsilon}=\Delta_{-\varepsilon} f(y) \approx \Delta_{\varepsilon} f(y),
$$

and thus, $\Delta_{\varepsilon^{\prime}} f(x) \approx \Delta_{\varepsilon} f(x) \approx \Delta_{\varepsilon} f(y) \approx \Delta_{\varepsilon^{\prime}} f(y)$, for all nonzero $\varepsilon^{\prime} \approx 0$.

Thus, we are ready to introduce the Heaviside function.

41. Definition. [Heaviside function] Let $f$ be differentiable on $(-1,1)$, with $f(x)=1$ for $x \geq 1$, $f(x)=0$ for $x \leq-1$, and $\int_{-1}^{1} f^{\prime}(x) d_{\pi} x \approx 1$. For $\varepsilon \approx 0$, the function $H_{\varepsilon}(x):=f(x / \varepsilon)$ is called a 'Heaviside function'.

We first consider two examples of Heaviside functions.

42. Example. The function $\mathfrak{H}_{\varepsilon}^{1}(x)\left(\right.$ resp. $\left.\mathfrak{H}_{\varepsilon}^{2}(x)\right)$ is defined as $\frac{x}{2 \varepsilon}+\frac{1}{2}\left(\right.$ resp. $\left.\frac{3}{2}\left(\frac{-x^{3}}{6 \varepsilon^{3}}+\frac{x}{2 \varepsilon}\right)+\frac{1}{2}\right)$ for $x \in[-\varepsilon, \varepsilon], 0$ for $x \leq-\varepsilon$ and 1 for $x \geq \varepsilon$.

We could require $f$ to be differentiable at 1 and -1 , but then we would exclude the functions $\mathbb{H}_{\varepsilon}^{1}(x)$ and $\mathbb{H}_{\varepsilon}^{2}(x)$ as Heaviside functions. Furthermore, the function $\mathbb{d}_{\varepsilon}^{1}(x)$ (resp. $\mathbb{d}_{\varepsilon}^{2}(x)$ ), defined as $\frac{1}{2 \varepsilon}$ (resp. $\left.\frac{3}{2}\left(\frac{-x^{2}}{2 \varepsilon^{3}}+\frac{1}{2 \varepsilon}\right)\right)$ if $x \in[-\varepsilon, \varepsilon]$ and 0 otherwise, is a delta function. If we ignore the points $\varepsilon$ and $-\varepsilon$, it is clear that $\Delta_{\varepsilon^{\prime}} \uplus_{\varepsilon}^{i}(x) \approx \mathbb{d}_{\varepsilon}^{i}(x)$ for $i=1,2$ and $\varepsilon^{\prime} / \varepsilon \approx 0$. Thus, we make the following notational convention.

43. Notation. For a Heaviside function $H_{\varepsilon}(x)$ and $\varepsilon^{\prime} / \varepsilon \approx 0$, we define $\Delta_{\varepsilon^{\prime}} H_{\varepsilon}(x)$, the derivative of $H_{\varepsilon}(x)$, as $\Delta_{\varepsilon^{\prime}} H_{\varepsilon}(x)$ if $x \in\left[-\varepsilon+2 \varepsilon^{\prime}, \varepsilon-2 \varepsilon^{\prime}\right]$ and 0 otherwise.

With this notation, we only ignore an interval that has infinitesimal length compared to $\varepsilon \approx 0$. Thus, the integral will not change more than an infinitesimal, even if $\Delta_{\varepsilon^{\prime}} H_{\varepsilon}(x)$ is infinite of magnitude $1 / \varepsilon$ around $\varepsilon$ and/or $-\varepsilon$. This notation is also motivated by the practice of measure theory, where an integral is not influenced by ignoring 'small' sets (i.e. sets of measure zero).

Now consider the following principle, called the 'Heaviside Theorem', or 'HEAT', for short. It states that the derivative of a Heaviside function is a delta function.

44. Principle (HEAT). Let $f$ be near-standard and continuous on $[a, b]$. If $\frac{|\pi|}{\varepsilon} \approx 0, \frac{\varepsilon^{\prime}}{\varepsilon} \approx 0$ and $a \ll 0 \ll b$, then

$$
\int_{a}^{b} \Delta_{\varepsilon^{\prime}} H_{\varepsilon}(x) \cdot f(x) d_{\pi} x \approx f(0) .
$$

45. Theorem. The Heaviside Theorem is provable in ERNA $+\Pi_{1}-$ TRANS. 
Proof. Let $f, \pi$ and $\varepsilon^{\prime}$ be as in the theorem and let $H_{\varepsilon}(x)=g(x / \varepsilon)$ be a Heaviside function. For $x \in\left[-\varepsilon+2 \varepsilon^{\prime}, \varepsilon-2 \varepsilon^{\prime}\right]$ and $\sigma:=\varepsilon^{\prime} / \varepsilon \approx 0$, we can calculate

$$
\frac{H_{\varepsilon}\left(x+\varepsilon^{\prime}\right)-H(x)}{\varepsilon^{\prime}}=\frac{g\left(\frac{x+\varepsilon^{\prime}}{\varepsilon}\right)-g\left(\frac{x}{\varepsilon}\right)}{\varepsilon^{\prime}}=\frac{1}{\varepsilon} \frac{g(x / \varepsilon+\sigma)-g(x / \varepsilon)}{\sigma}=\frac{1}{\varepsilon} \cdot \Delta_{\sigma} g(x / \varepsilon) .
$$

By definition, the function $\Delta_{\sigma} g(x)$ is integrable over $[-1,1]$. Now apply Theorem 34 with $a=$ $-\varepsilon+2 \varepsilon^{\prime}$ and $b=\varepsilon-2 \varepsilon^{\prime}$. Thus, $\frac{1}{\varepsilon} \Delta_{\sigma} g(x / \varepsilon)$ is $\varepsilon$-integrable over $\left[-\varepsilon+2 \varepsilon^{\prime}, \varepsilon-2 \varepsilon^{\prime}\right]$, and, for $\eta:=\frac{2 \varepsilon^{\prime}}{\varepsilon} \approx 0$, we have

$$
\int_{-\varepsilon+2 \varepsilon^{\prime}}^{\varepsilon-2 \varepsilon^{\prime}} \Delta_{\sigma} g(x / \varepsilon) 1 / \varepsilon d_{\pi} x \approx \int_{-1+\eta}^{1-\eta} \Delta_{\sigma} g(x) d_{\rho} x \approx 1 .
$$

Using ERNA's Weierstrass Extremum principle, the theorem follows in the same way as for Theorem 37. Alternatively, apply Theorem 34 to the integrand $\Delta_{\varepsilon^{\prime}} H_{\varepsilon}(x) \cdot f(x)$, yielding $\int_{-1+\eta}^{1-\eta} \Delta_{\sigma} g(x)$. $f(\varepsilon x) d_{\pi} x$. The latter is clearly infinitely close to $f(0)$.

Finally, we have the following theorem.

\section{Theorem. In ERNA, HEAT is equivalent to $\Pi_{1}$-TRANS.}

Proof. The reverse direction follows immediately from Theorem 45 . The forward direction is proved in the same way as in Theorem 38

Principle 44 contains the conditions $\frac{|\pi|}{\varepsilon} \approx 0$ and $\frac{\varepsilon^{\prime}}{\varepsilon} \approx 0$. If we wanted to treat $n$-th order derivatives of suitable $H_{\varepsilon}(x)$, we would require $n+1$ such conditions. Similarly, for suitable delta functions $\delta_{\varepsilon}(x)$, we could consider $\Delta_{\varepsilon^{\prime}} \delta_{\varepsilon}(x)$ for $\varepsilon^{\prime} / \varepsilon \approx 0$ and higher-order derivatives. This would result in the property $\int_{a}^{b} \delta_{\varepsilon}^{(n)}(x) \cdot f(x) d_{\pi} x \approx(-1)^{n} f^{(n)}(0)$. From the above, it is clear that the latter formula gives rise to theorems equivalent to $\Pi_{1}$-TRANS. However, the amount of technical detail becomes unmanageable, suggesting the need for a more suitable framework. For ERNA, such a framework was introduced in [13, where infinitesimals of different magnitude are built into the theory ERNA. This fundamental insight goes back to Péraire and Hrbacek (7, 11]).

\section{REFERENCES}

[1] Douglas K. Brown, Mariagnese Giusto, and Stephen G. Simpson, Vitali's theorem and WWKL, Arch. Math. Logic 41 (2002), no. 2, 191-206.

[2] Rolando Chuaqui and Patrick Suppes, Free-variable Axiomatic Foundations of Infinitesimal Analysis: A Fragment with Finitary Consistency Proof, Journal of Symbolic Logic 60 (1995), 122-159.

[3] Paul A. M. Dirac, The Principles of Quantum Mechanics, Oxford, at the Clarendon Press, 1927.1 st ed.

[4] Harvey Friedman, Some systems of second order arithmetic and their use, Proceedings of the International Congress of Mathematicians (Vancouver, B. C., 1974), Vol. 1, Canad. Math. Congress, Montreal, Que., 1975, pp. $235-242$.

[5] _ Systems of second order arithmetic with restricted induction, I \& II (Abstracts), Journal of Symbolic Logic 41 (1976), 557-559.

[6] Harvey M. Friedman, Stephen G. Simpson, and Rick L. Smith, Countable algebra and set existence axioms, Ann. Pure Appl. Logic 25 (1983), no. 2, 141-181.

[7] Karel Hrbacek, Relative Set Theory: Internal View, Journal of Logic and Analysis 1 (2009), no. 8, 1-108.

[8] Chris Impens and Sam Sanders, The strength of nonstandard analysis (Imme van den Berg and Vítor Neves, eds.), Springer Wien New York Vienna, 2007.

[9] _ _ Transfer and a supremum principle for ERNA, Journal of Symbolic Logic 73 (2008), 689-710.

[10] _ Saturation and $\Sigma_{2}$-transfer for ERNA, Journal of Symbolic Logic 74 (2009), 901-913.

[11] Yves Péraire, Théorie relative des ensembles internes, Osaka J. Math. 29 (1992), no. 2, 267-297 (French).

[12] Nobuyuki Sakamoto and Takeshi Yamazaki, Uniform versions of some axioms of second order arithmetic, MLQ Math. Log. Q. 50 (2004), no. 6, 587-593.

[13] Sam Sanders, More infinity for a better finitism, Annals of Pure and Applied Logic 121 (2010), 1525-1540. 
[14] _ ERNA and Friedman's Reverse Mathematics, Journal of Symbolic Logic 76 (2011).

[15] Laurent Schwartz, Théorie des distributions, Hermann, Paris, 1951.

[16] Stephen G. Simpson, Which set existence axioms are needed to prove the Cauchy/Peano theorem for ordinary differential equations?, J. Symbolic Logic 49 (1984), no. 3, 783-802.

[17] Stephen G. Simpson and Keita Yokoyama, A nonstandard counterpart of WWKL. preprint.

[18] Stephen G. Simpson, Subsystems of second order arithmetic, 2nd ed., Perspectives in Logic, Cambridge University Press, Cambridge, 2009.

[19] _ (ed.), Reverse mathematics 2001, Lecture Notes in Logic, vol. 21, Association for Symbolic Logic, La Jolla, CA, 2005.

[20] Richard Sommer and Patrick Suppes, Finite Models of Elementary Recursive Nonstandard Analysis, Notas de la Sociedad Mathematica de Chile 15 (1996), 73-95.

[21] Patrick Suppes and Rolando Chuaqui, A finitarily consistent free-variable positive fragment of Infinitesimal Analysis, Proceedings of the IXth Latin American Symposium on Mathematical Logic Notas de Logica Mathematica 38 (1993), 1-59.

[22] Keita Yokoyama, Standard and non-standard analysis in second order arithmetic, $\mathrm{PhD}$ thesis, Tohoku University, Sendai, 2007. Available as Tohoku Mathematical Publications 34, 2009.

[23] Xiaokang Yu and Stephen G. Simpson, Measure theory and weak König's lemma, Arch. Math. Logic 30 (1990), no. $3,171-180$. 